\title{
Investigating the stability of and relationships among global/local processing measures
}

\author{
Gillian Dale • Karen M. Arnell
}

Published online: 26 January 2013

(C) Psychonomic Society, Inc. 2013

\begin{abstract}
Global/local stimuli have been used to estimate global processing biases in individuals and groups, as well as in response to various manipulations. Throughout the literature, multiple different versions of global/local stimuli have been used, such as traditional hierarchical letters and numbers (i.e., Navon letters), abstract hierarchical shapes, and high- and low-spatial-frequency gratings and faces. However, currently it is unclear how reliable or stable performance is on these measures within individuals over time, and whether these seemingly different measures are tapping into the same underlying process. As such, the purpose of the present study was to examine the stability of individual performance on three distinct global/local measures over time and to examine the relationships among the measures. In two studies, we examined the reliability of the biases within, and the relationships among, standard Navon letters in a traditional interference task, hierarchical shapes in a forced choice task, and superimposed high- and low-pass spatial frequency faces in a forced choice task. In both studies, participants completed all three of the tasks, and then returned 7-10 days later to again complete the same tasks. The degree of global/local bias within an individual was found to be highly reliable in the hierarchical shape task and the spatial frequency face task, but less reliable in the traditional Navon letter task. Interestingly, in both studies we found that none of the three measures of global bias were related to each other. Therefore, while these measures do appear to be reliable over time, they may be tapping into distinct aspects of global/local processing.
\end{abstract}

\section{G. Dale $\cdot$ K. M. Arnell $(\bowtie)$}

Department of Psychology, Brock University,

500 Glenridge Avenue,

St. Catharines, ON L2S 3A1, Canada

e-mail: karnell@brocku.ca
Keywords Global/local bias · Global precedence · Individual differences $\cdot$ Reliability

Visual stimuli can often be viewed at either a broad, global level (e.g., the forest) or at a more detailed, local level (e.g., a tree). Researchers often investigate a bias toward global or local information with hierarchical global/local stimuli known as "Navon stimuli" (Navon, 1977, 1981). Navon stimuli are typically large single letters that are composed of smaller letters (see Fig. 1c below and Navon, 1977). Variations of such stimuli can also involve hierarchical shapes (Kimchi \& Palmer, 1982) or objects (Fink et al., 1997).

For Navon stimuli, the large element represents the global perceptual level, whereas the smaller elements represent the local perceptual level. The elements at the two different levels can either be the same (congruent) or different (incongruent). Participants are usually directed to attend to either the global or the local level and to identify the stimulus at that level as quickly as possible. The results from using hierarchical stimuli typically show that more interference occurs from the global information when one is trying to focus on the local information than occurs in the reverse situation (i.e., a global advantage). This suggests that the processing of the broad aspects of a stimulus takes precedence over the processing of finer, more detailed aspects (Navon, 1981).

\section{Individual differences}

While a global advantage is generally observed with Navon stimuli, evidence suggests that the degree of individual bias toward global information can be altered by varying stimulus parameters, such as the aspect ratio of the local to the global items (Kimchi, 1992; Yovel, Yovel, \& Levy, 2001), the overall visual angle (Kinchla \& Wolfe, 1979), or the exposure 


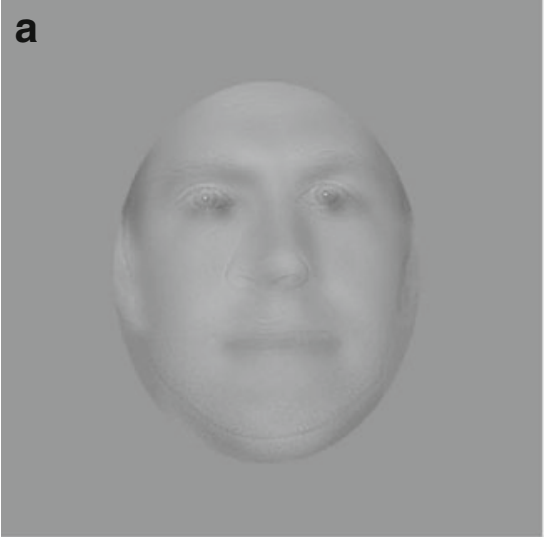

b
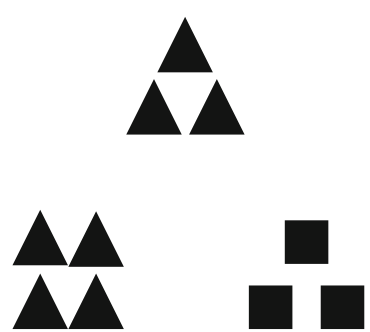

C

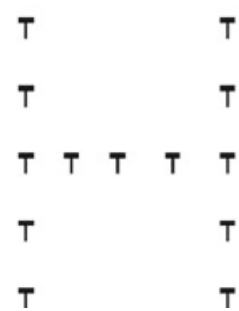

Fig. 1 (a) Sample hybrid face stimulus from a global/local face task. After the hybrid face is presented, it is replaced on the screen by the two faces that composed the hybrid, in their unfiltered forms. Participants select the unfiltered face that they believe best matches the previously viewed hybrid face. (b) Sample test shape triad from the global/local shape task. Participants are required to select the one of the bottom two figures in the triad that they believe best matches the top figure. (c) Sample incongruent Navon letter from the Navon letter task. Participants are directed to indicate the identity of the letter at either the local or the global level, as quickly as possible

duration (Paquet \& Merikle, 1984). Kimchi and Palmer (1982), in particular, have shown that the relative number and size of the elements in a global/local hierarchical figure can greatly influence whether or not a global advantage emerges. Specifically, the use of relatively few, large-size local elements within the global pattern promotes a local processing advantage, while the use of many smaller local elements within the global pattern promotes a global processing advantage.

In addition, individuals have been shown to vary widely in terms of their degrees of dispositional global or local bias. Some individuals have a natural bias for global information, some have a natural bias for local information, and some show little to no bias (Dale \& Arnell, 2010). A variety of participant characteristics affect the degrees of individual global or local bias. For example, older individuals (e.g., Lux, Marshall, Thimm, \& Fink, 2008), individuals induced into a state of negative affect (Gasper \& Clore, 2002), individuals from remote cultures (Davidoff, Fonteneau, \& Fagot, 2008), and musicians (Stoesz, Jakobson, Kilgour, \& Lewycky, 2007) all appear to show a larger local than global bias. Conversely, individuals from collectivist cultures show more of a global bias (McKone et al., 2010), as do individuals who have been induced into a positive affective state (Fredrickson \& Branigan, 2005). Thus, a global advantage is not apparent in all individuals, nor is it absolute.

One way in which these individual differences in global/ local bias can be captured is through the use of neutral global/local stimuli, in which both the global and local levels are equally salient (Fredrickson \& Branigan, 2005). While typically a global advantage emerges, using a sparser hierarchical display (i.e., fewer local elements) or equating the salience of the figures can allow for greater variability in responses (Fredrickson \& Branigan, 2005; Kimchi \& Palmer, 1982), thus effectively capturing natural differences in individual global/local bias. Using this rationale, Dale and Arnell (2010) showed that individual differences in dispositional global bias, as assessed by a Navon letter interference task, predicted dual-task attention costs in the attentional blink paradigm, in that greater global bias was associated with smaller attentional blinks. Martin and Macrae (2010) have also estimated individual differences in global processing bias using a global/local Navon letter task, and they showed that when individuals with a large global bias performed a face recognition task, they produced a larger face inversion effect - thought to reflect the degree of holistic face processing - than did individuals with weak global bias.

Despite the fact that researchers have begun to use global/ local bias as an individual-differences variable, we have yet to determine whether an individual's global/local preference is a stable trait that persists over time. Therefore, the primary purpose of the present article was to examine the stability of performance over time on three very distinct global/local tasks, in order to determine whether individual global/local bias is a stable individual-differences variable.

In addition to the popular computerized Navon letter task (Navon, 1981) discussed above, two other tasks were included that have also been used to assess differences in global/local processing bias across groups. One of these was a paper-and-pencil hierarchical shape task adapted from Kimchi and Palmer (1982) and Fredrickson and Branigan (2005). In this task, participants were shown a hierarchical "standard" figure in which the global shape was made up of several local shapes (e.g., a square made of triangles). The participants were then asked to choose which of two "comparison" hierarchical figures (squares or triangles) best matched a standard figure. One of the comparison figures 
matched the standard at the global level, and one at the local level (see Fig. 1b). This task has been used to show that both the number and size of the local elements in a figure can influence the magnitude of the global/local advantage (Kimchi \& Palmer, 1982). It has also been used to show that induced state affect can modulate the global/local processing advantage, such that positive states promote a global processing bias (Fredrickson \& Branigan, 2005) and negative states promote a local processing bias (Gasper \& Clore, 2002).

In a third task, hierarchical stimuli were not used. Instead, two faces of different individuals were displayed superimposed on the computer screen. One face contained only high-spatial-frequency information, and the other face contained only low-spatial-frequency information. Participants were then shown both unfiltered faces and asked to choose which of the two faces had just been presented. Using this task, Deruelle, Rondan, SalleCollemiche, Bastard-Rosset, and Da Fonséca (2008) showed that when matching faces for identity or emotion, children with autistic spectrum disorders showed a greater preference for local information than did control children.

As noted above, performance on hierarchical stimulus tasks often shows a global processing advantage. However, the direction (global or local) and degree of the advantage can be modulated by changes in the relative size and number of local elements relative to the global shape (Kimchi, 1992; Kimchi \& Palmer, 1982). Following the rationale of Fredrickson and Branigan (2005), pilot testing and the existing literature were used to create stimuli that were global/local neutral (i.e., stimuli likely to induce no overall global/local advantage in the sample as a whole), so that the natural inclinations of the participants would not be enhanced or countered by stimuli that promoted a particular bias.

In addition to examining the reliability of our measures, we were also interested in examining the relationships among our three distinct global/local processing tasks. Two of the three global/local processing tasks that we selected have previously been used as indexes of general global/local processing bias, whereas the third task is a spatial frequency task that is associated with global/local processing. Various global/local measures have been used as if they measured the same thing; however, the degree to which each of these tasks relates to each of the others is currently unknown.

\section{Study 1}

Method

\section{Participants}

Sixty Brock University undergraduate students (56 women) ranging in age from 17 to 33 years $(M=19.6)$ voluntarily participated in this study. All of the participants reported learning English before the age of 8 and reported normal or corrected-to-normal vision. Participants completed a 1$\mathrm{h}$ testing session, followed by a second 1 -h session 7-10 days later. A total of five individuals did not return for the second session and were thus removed from the analyses, leaving a total of 55 participants. All participants completed a global/ local face task first, followed by a global/local paper task, and then a global/local Navon letter task.

\section{Apparatus}

The computerized tasks were presented using E-Prime software on a Dell desktop computer with a dual-core processor and a 17-in. CRT monitor. All responses in the computer tasks were made via buttonpress on the computer keyboard.

\section{Stimuli and design}

Global/local face task This task was adapted from Deruelle et al. (2008). A set of 27 male and 27 female normed young adult faces with neutral expressions and no facial hair were obtained from the Center for Vital Longevity Face Database (Minear \& Park, 2004). The faces were cropped to remove head hair, converted to grayscale, and pasted onto a $480 \times$ 480 pixel dark gray background so that they subtended approximately $16^{\circ}$ of visual angle with an unrestrained viewing distance of approximately $55 \mathrm{~cm}$. High-pass and low-pass spatial frequency faces were then constructed in Adobe Photoshop from the original 54 faces (one high and one low for each face). High-pass-filtered faces were constructed by using a high-pass filter in Photoshop, so that they contained only spatial frequencies higher than 6 cycles/ degree of visual angle (i.e., a radius of 1.5 pixels). ${ }^{1}$ Lowpass-filtered faces were constructed by using a Gaussian blur in Photoshop, so that they contained only spatial frequencies lower than 2 cycles/degree of visual angle (i.e., a radius of 4.5 pixels). High-/low-pass hybrid faces were then created by superimposing the high-pass face of one person over the low-pass face of another person (matched for gender). The high- and low-pass-filtered faces were equated for luminance and size, and they were roughly equal in salience. A total of 54 hybrid faces were constructed, with each original face contributing high-frequency information to one hybrid face and low-frequency information to another hybrid face (see Fig. 1a).

Each trial began with a 1,000-ms fixation cross, after which a hybrid face appeared in the center of the screen for $300 \mathrm{~ms}$. It was then replaced by the two original

\footnotetext{
${ }^{1}$ To convert Adobe Photoshop radius into cycles/degree, we used the following formulas: $\tan ^{-1}$ (radius/viewing distance) or $\tan ^{-1}$ (PPC/viewing distance).
} 
(unfiltered) faces that comprised the hybrid face (i.e., one intact face whose high-frequency information had been used in the hybrid, and another intact face whose low-frequency information had been used in the hybrid). One of the intact faces was presented on the left side of the screen, and the other was on the right (counterbalanced). Participants were asked to select the original face that they thought best matched the hybrid face by pressing the corresponding key on the keyboard. These faces remained on the screen until the participant made a response. Responses were not speeded, but the participants were encouraged to go with their first instinct. Each participant performed 54 trials. For each participant, a global face score was calculated as the total number of trials out of 54 on which the participant selected the face whose low-frequency information had been used in the face hybrid. Therefore, a high global-face score would suggest a bias for global processing, whereas a low globalface score would suggest a bias for local processing.

Global/local paper task Participants were presented with a booklet that contained global/local shape triads, adapted from those of Kimchi and Palmer (1982) and Fredrickson and Branigan (2005). Shape triads were composed of three hierarchical shapes, arranged with a standard figure on top and two comparison figures on the bottom (see Fig. 1b). In each case, participants were required to circle the comparison figure that they felt best matched the standard figure. They were instructed to use their first instinct and to proceed as quickly as possible.

Over the trials, eight test triads and 16 filler triads were intermixed, for a total of 24 triads. The hierarchical shapes in each test triad consisted of 3-4 small $(5 \times 5 \mathrm{~mm})$ square or triangle shapes (local level) that formed a larger $(15 \times$ $15 \mathrm{~mm}$ ) square or triangle (global level). For the test triads, both of the comparison figures matched the standard figure, but one matched at the global level (the overall shape outline matched the standard), and one matched at the local level (the smaller shape matched the standard). The hierarchical shapes in each filler triad were composed of 3-4 small $(5 \times$ $5 \mathrm{~mm}$ ) circles, squares, triangles, or crosses (local level) that formed a larger $(15 \times 15 \mathrm{~mm})$ square or triangle (global level). We chose sparse hierarchical figures in order to better detect individual differences in global/local bias, as per Fredrickson and Branigan (2005).

After completion of the task, the total number of test triads in which the global comparison shape was selected was calculated for each participant, resulting in a global score that could range from 0 to 8 . Therefore, a high total would reflect a global bias, and a low total would reflect a local bias. Filler triads had only one correct response (half with global correct, and half with local correct); thus, they were not used as indexes of global/ local bias.
Global/local Navon letter task Each trial began with a 500ms central fixation cross, after which a single Navon stimulus was presented in the center of the computer screen. The Navon stimuli were large letters constructed of smaller letters (e.g., an "H" made out of "T"s; see Fig. 1c.). The global letters $(70 \times 50 \mathrm{~mm})$ were ten times as large as the local letters $(7 \times 5 \mathrm{~mm})$. The viewing distance was approximately $55 \mathrm{~cm}$, unrestrained. Pilot testing suggested that the global and local levels were roughly equal in salience. All letters appeared in black New Courier font on a white background. The letters that were presented could be either "H" or "T." Half of the trials in each condition were congruent (i.e., an "H" made of small " $H$ "s or a "T" made of small "T"s) and half were incongruent (an "H" made of small "T"s or a "T" made of small "H"s), and these were randomly mixed within each block. Global and local trials were presented in alternating blocks, with 24 trials in each of four blocks, for a total of 96 trials. All participants began with the global block. ${ }^{2}$ Participants were required to quickly report either the identity of the smaller letters (local trials) or the identity of the large letter (global trials) by pressing the corresponding key on the keyboard. The stimulus remained on the screen until the participant had made a response.

RTs were examined for each combination of participant, task (global/local report), and condition (congruent/incongruent). RTs for incorrect trials or that fell outside three standard deviations from the mean were removed. Global and local interference, along with mean global and local RTs, were then calculated for each participant. Local interference was calculated as the degree to which local features influenced performance on the global trials (global incongruent RT - global congruent RT), and global interference was measured as the degree to which global features influenced performance on the local trials (local incongruent RT - local congruent RT).

\section{Results}

\section{Face task}

The mean Session 1 global face score was $29.75(S D=$ 5.93), and the mean Session 2 global face score was 29.44 $(S D=7.33)$ out of 54 . This indicates that just over half of the trials were classified at the global level in each session.

\footnotetext{
${ }^{2}$ All participants completed the Navon letter task blocks in the same order, so that estimates of the participants' global and local interference would not be confounded with block order. When conducting an individual-differences study, it is not ideal to counterbalance the tasks or blocks across participants, as performance on tasks/blocks may differ somewhat because of the order in which they are presented. Therefore, a participant's relative score on a given task could be confounded with order variability if the order was counterbalanced or random. This confound can be removed in individual-differences studies by using a constant task order.
} 
The slight global advantage was significant statistically in each session $(p s<.018)$ as compared to the chance performance of 27; however, the small size of the differences from 27 suggests that the stimuli did not greatly bias the participants overall into choosing the global or the local face. We found large individual differences in task performance, with average scores across sessions ranging from 17 to 45 out of 54.

\section{Paper task}

The mean Session 1 global paper score was $3.36(S D=2.21)$, and the mean Session 2 global paper score was $4.11(S D=$ 2.51 ) out of 8 . As compared to a score of 4 , which would reflect chance performance, mean scores across the participants showed a small, but significant, local bias in Session 1, $t(54)=2.14, p=.037$, but no bias in Session $2(p=.74)$ or overall across sessions $(p=.38)$, suggesting that the stimuli left lots of room for dispositional differences in global/local bias to emerge. Indeed, large individual differences in task performance did emerge, with average scores across sessions ranging from 0 to 7.5 out of 8 .

\section{Navon letter task}

The mean letter identification RTs for Sessions 1 and 2 of the computerized Navon letter task are presented in Fig. 2a and b, as a function of global or local task block and the congruence/incongruence of the global and local levels. Mean RTs were analyzed using a $2 \times 2$ (Task $\times$ Congruency) repeated measures analysis of variance (ANOVA).

For Session 1, we found a significant main effect of congruency, with faster RTs on congruent than on incongruent trials, $F(1,54)=35.93 p<.001$. No significant main effect of global/local block was apparent, $F<1$. Additionally, the interaction between feature size and congruency was not significant, indicating that local and global interference were equal in magnitude, $F<1$.

For Session 2, we again found a significant main effect of congruency, with faster RTs on congruent than on incongruent trials, $F(1,54)=39.77, p<.001$. We also found a significant main effect of stimulus feature level, in which RTs were faster on global than on local trials, $F(1,54)=11.34, p=.001$. Once again, the interaction between feature size and congruency was not significant, indicating that the local and global forms of interference were equal in magnitude, $F<1$. Therefore, as intended, the stimuli did not bias the participants, as a group, toward global or local processing, leaving lots of room for dispositional variation in global/local bias. Indeed, across sessions the average global interference scores ranged from -95 to $144 \mathrm{~ms}$.
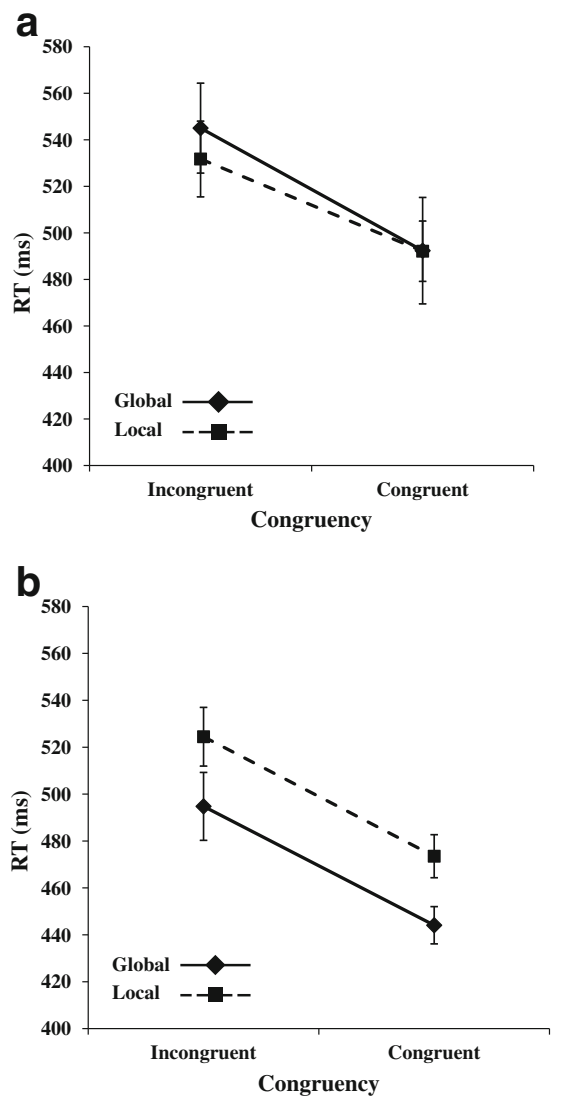

Fig. 2 (a) Mean Session 1 RTs on the Navon letter task, as a function of task (attend global or attend local) and target congruency. Error bars represent the standard errors for each condition mean. (b) Mean Session 2 RTs on the Navon letter task, as a function of task (attend global or attend local) and target congruency. Error bars represent the standard errors for each condition mean

The mean error rates in the Navon letter task were $5 \%$ and $4 \%$ for Sessions 1 and 2, respectively. A $2 \times$ 2 (Congruency $\times$ Global/Local Task) repeated measures ANOVA on the mean error data for each session showed that errors were greater for incongruent than for congruent trials, $F(1,54)=36.91, p<.001$, and $F(1,54)=42.10, p<.001$, for Sessions 1 and 2, respectively. No main effect of stimulus level appeared, nor an interaction of congruence and stimulus level for either session, all $F \mathrm{~s}<1$.

\section{Test-retest reliability}

As an index of test-retest reliability, the Pearson correlation coefficients between scores on Session 1 versus Session 2 were examined individually for each of the three global/ local tasks. Global/local bias on the face global/local task was shown to be highly reliable over time, as was the bias on the paper global/local task (see the bolded values in Table 1, as well as Fig. 3a, b, and c). The test-retest reliability of the Navon letter task, however, was quite low, 
Table 1 Pearson zero-order correlations between test sessions for the three global-local measures (in bold font), as well as the relationships among all of the measures in Study 1

\begin{tabular}{lcccccc}
\hline & 1 & 2 & 3 & 4 & 5 \\
\hline 1. Global face, Session 1 & - & & & & \\
2. Global shape, Session 1 & .12 & - & & & \\
3. Global letter interference, Session 1 & -.10 & .11 & - & & \\
4. Global face, Session 2 & $.70^{* *}$ & -.14 & -.05 & - & \\
5. Global shape, Session 2 & .17 & $.79^{* * *}$ & .02 & -.01 & - \\
6. Global letter interference, Session 2 & -.19 & .10 & $\mathbf{. 3 1}^{*}$ & -.08 & .14
\end{tabular}

${ }^{*} p<.05,{ }^{* *} p<.01$. Bold font indicates test-retest correlations

albeit significant. When we examined the mean Navon letter global and local RTs across sessions, both had high testretest reliability ( $r=.66$ and .73 , respectively). This suggests that while the RTs were highly reliable, the measure of interference was not. ${ }^{3}$

\section{Relationships among the measures}

The relationships among the three different measures of global/local processing bias were examined by correlating the scores for each test session. Interestingly, none of the measures were significantly related to each other, either within a session or across sessions (see the nonbolded values in Table 1). When the scores for each of the three tasks were combined across the two sessions, we once again found no significant relationship among the three measures (all $p \mathrm{~s}>.33$; see Fig. $4 \mathrm{a}, \mathrm{b}$, and c).

\section{Factor analysis}

In order to further explore the independence of our three measures, we performed a factor analysis with a varimax rotation that included our three measures of global bias for each of the two sessions (six items). We obtained a threefactor solution, ${ }^{4}$ with each of the three global/local tasks loading on its own factor (see Table 2).

\footnotetext{
${ }^{3}$ One may be concerned that the relatively poor reliability of the Navon letter task resulted solely from the use of a difference score to estimate global interference. However, almost the same reliabilities were observed when incongruent local RTs on Session 1 were used to predict incongruent local RTs on Session 2, with the variability from local congruent RTs partialed out for each session $(r=.30$ in Study 1 and $\mathrm{r}=.20$ in Study 2).

${ }^{4}$ To determine the correct number of components to retain in our factor analysis, we first used the Kaiser criterion method, also called the "eigenvalue-one criterion." This method retains only those factors that have eigenvalues greater than 1 (Nunnally, 1978). With this method, an obvious three-factor solution was apparent. Additionally, when we performed a scree test, as recommended by Cattell (1966), once again an obvious three-factor solution emerged.
}

\section{Discussion}

The primary goal of Study 1 was to examine the stability of individual differences in global/local bias over time. The results indicated that three distinct measures of dispositional global/local bias were reliable over the period of 7-10 days, with two of the tasks showing high reliability. This suggests that individual differences in global/local bias or preference are relatively stable over time and supports their use in studies of individual differences. However, while the face and shape tasks were highly reliable, performance on the Navon letter task had fairly low reliability over time, suggesting that this task may not be accurately measuring global/local processing. This is problematic, considering the popularity of this particular measure.

In addition to examining the reliability of our tasks, we were interested in examining how they were interrelated. Surprisingly, while the three tasks were reliable, none of them related to each other, either within or across sessions, or when the data from both sessions were combined. In addition, when a factor analysis was performed, we found that the three tasks loaded onto unique factors, suggesting that the tasks do not share an underlying construct. This indicates that while the three tasks may still be measuring global/local processing bias, they may be measuring different aspects of this construct. This finding is particularly alarming, given that the letter and shape tasks are often used interchangeably as an index of global/local processing.

However, the stimuli used in each of the tasks were created in such a way as to promote neither a global nor a local bias, so as to better capture individual differences without having the stimuli themselves constrain the range of individual differences by biasing individuals in a given direction. As such, two of the three tasks (the Navon letter task and the hierarchical shape task) did not show a typical global advantage. One might be concerned that the lack of global advantage in our tasks, and the presentation conditions required in order to produce global/local neutral stimuli, may have changed what the tasks themselves were measuring. This could potentially have led to the lack of relationship among our tasks, and might also have led to the low reliability of the traditional Navon letter task. As such, we conducted Study 2, which used stimuli designed to promote a more typical global bias. The purpose of Study 2 was the same as in Study 1, such that we were interested in examining whether the results for three distinct global/local tasks would be reliable over time, and whether performance would be related across these tasks. 

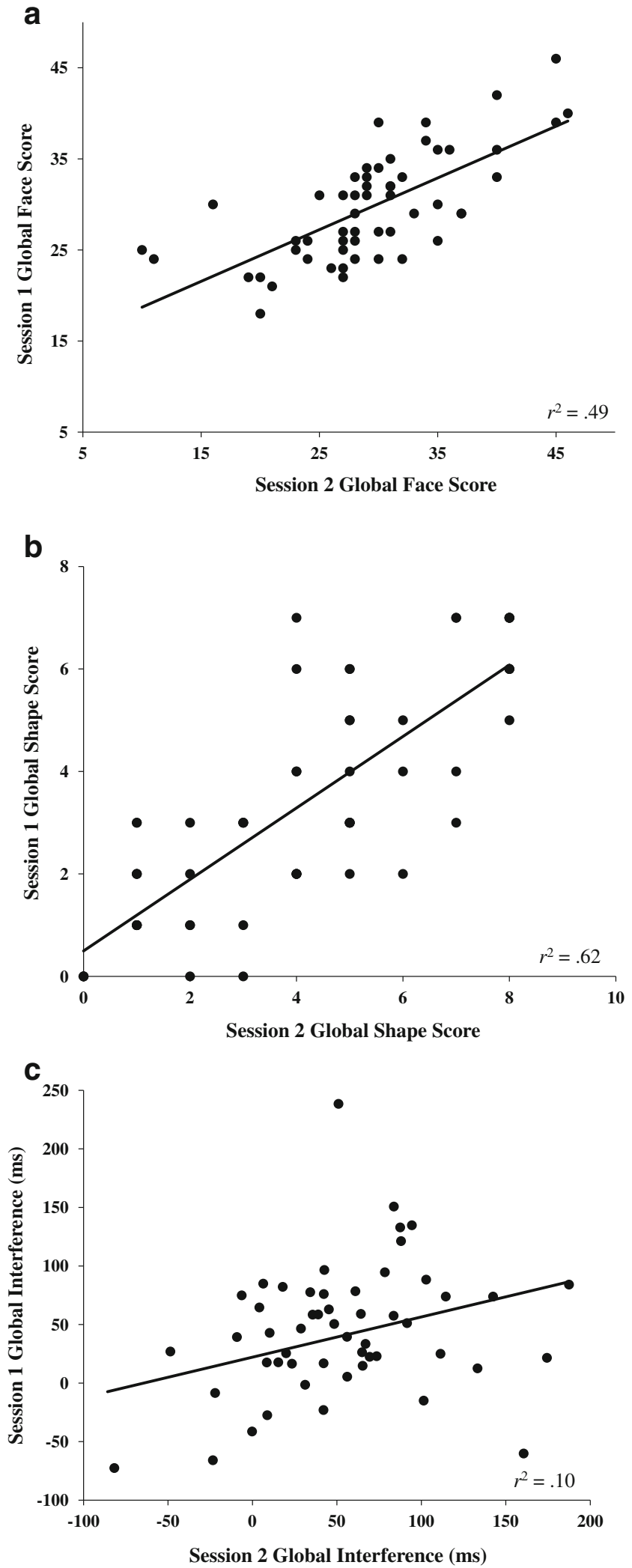

Fig. 3 (a) Scatterplot depicting Pearson $r$ correlations between Session 1 and 2 scores on the global/local face task. (b) Scatterplot depicting Pearson $r$ correlations between Session 1 and 2 scores on the global/local shape task. (c) Scatterplot depicting Pearson $r$ correlations between Session 1 and 2 scores on the global/local Navon letter task
Study 2

Method

\section{Participants}

Fifty-eight Brock University undergraduate students (47 women, 11 men) ranging in age from 18 to 30 years $(M=$ 19.7) voluntarily participated in this study and had the same restrictions as in Study 1. Participants completed a 1-h testing session, followed by a second 1 -h session $7-10$ days later. All of the participants completed the hierarchical shape task first, followed by the global/local face task, and then the global/ local Navon letter task. All of these tasks were the same as in Study 1, but with the following alterations.

\section{Stimuli and design}

Global/local face task For the global/local face task, the duration of the hybrid face was decreased from 300 to $150 \mathrm{~ms}$. This was done in order to make the low-spatialfrequency face more salient for participants (Paquet \& Merikle, 1984). Additionally, the number of faces used was decreased from 27 to 21 , such that 42 hybrid pairs were now presented. This was done to remove the six faces that had resulted in almost exclusively global or local responses in Study 1, and thus might have limited the variability in this measure. As such, the total global face score was now out of 42.

Hierarchical shape task For Study 2, the pen-and-paper shape task was converted into a computerized task. This was done in order to control the viewing time for each of the stimuli, as opposed to the virtually unlimited viewing time that participants had had for the stimuli in Study 1. Some of the variability in this task in Study 1 might have resulted from individual differences in how long the participants chose to examine the stimuli before responding. This new computerized version equated participants on this measure.

Eight different hierarchical shapes were constructed, such that each hierarchical shape contained both a global and a local level. Each of the hierarchical shapes was composed of 15-25 small $(2 \times 2 \mathrm{~mm})$ circles, squares, triangles, or crosses (local level) that formed a larger $(20 \times 20 \mathrm{~mm})$ square or triangle (global level). Note that these shapes were denser than those used in the previous study, as density has previously been shown to increase global saliency (e.g., Kimchi \& Palmer, 1982).

These hierarchical shapes were then grouped into triads, such that each triad contained three of the hierarchical shapes. In each triad, one of the hierarchical shapes was designated the "standard" shape, and the other two were called the "comparison" shapes. For half 

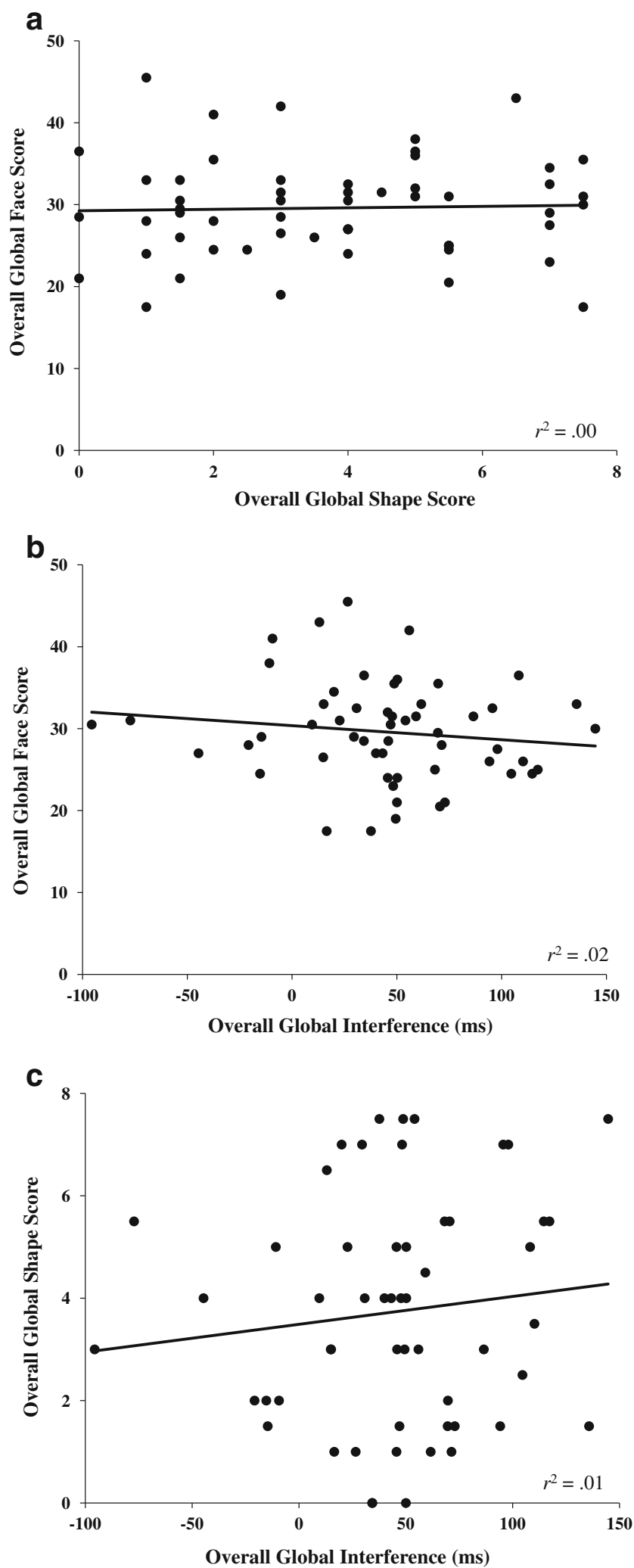

Fig. 4 (a) Scatterplot depicting Pearson $r$ correlations between overall global face task score and overall global shape task score, $r=.03$. (b) Pearson $r$ correlations between overall global face task score and overall global interference from the letter Navon task, $r=-.13$. (c) Pearson $r$ correlations between overall global shape task score and overall global interference from the letter Navon task, $r=.12$

of the triads, one of the comparison shapes matched the standard shape at the global level, and the other comparison shape matched the standard at the local level (a.k.a. the "test" triads). For the other half of the triads, only one of the comparison shapes matched the standard at either the global or the local level (a.k.a. the "filler" triads). In total, 16 test triads and 16 filler triads were intermixed, for a total of 32 triads.

Each trial began with a $1,000-\mathrm{ms}$ blank screen, after which the standard hierarchical shape appeared in the center of the screen for $50 \mathrm{~ms}$. This standard shape was then replaced by the two comparison hierarchical shapes. One of the shapes was presented on the left side of the screen and one on the right (counterbalanced). Participants were asked to select the comparison shape that they thought best matched the standard shape by pressing the corresponding key on the keyboard. These comparison shapes remained on the screen until the participant made a response. Responses were not speeded, but participants were encouraged to go with their first instinct. Each participant performed 32 trials. For each participant, a global shape score was calculated as the total number of test trials out of 16 in which the participant selected the comparison shape that matched the standard at the global level. Therefore, a high global shape score would suggest a bias for global processing, whereas a low global score would suggest a bias for local processing. The filler triads had only one correct response (half with global correct, and half with local correct), and thus were not used as indexes of global/local bias.

Global/local Navon letter task The Navon letter task was basically the same as in Study 1, with the exception that the density of the letters was increased by using more local letters (roughly 25 letters, as opposed to 13). Additionally, the letter stimuli now only appeared on the screen for a

Table 2 Rotated factor loadings for the three global/local measures, by sessions, showing three distinct factors in Study 1

\begin{tabular}{|c|c|c|c|}
\hline & \multicolumn{3}{|c|}{ Component } \\
\hline & 1 & 2 & 3 \\
\hline Global face, Session 1 & .17 & .91 & -.14 \\
\hline Global face, Session 2 & -.11 & .93 & .01 \\
\hline Global shape, Session 1 & .95 & -.03 & .07 \\
\hline Global shape, Session 2 & .94 & .07 & .05 \\
\hline Global letter interference, Session 1 & .01 & .01 & .82 \\
\hline Global letter interference, Session 2 & .09 & -.12 & .79 \\
\hline
\end{tabular}

Method: Principal components analysis, varimax with Kaiser normalization 
duration of $15 \mathrm{~ms}$, after which the stimuli were replaced with a blank screen, which remained until participants made a response. Increasing the density of the display typically makes the stimuli more globally salient (e.g., Kimchi \& Palmer, 1982), and reducing the display time of the letter stimuli themselves was also expected to increase the saliency of the global stimuli, on the basis of a similar effect reported by Paquet and Merikle (1984).

Results

\section{Global/local face task}

The mean Session 1 global face score was $24.45(S D=$ 5.54), and the mean Session 2 global face score was 23.79 $(S D=4.67)$ out of 42 . This indicates that just over half of the trials were classified at the global level in each session. This global advantage was significant statistically in each session $(p s<.001)$ as compared to chance performance of 21. Large individual differences in task performance emerged, with average scores across sessions ranging from 11 to 38 out of 42 .

\section{Hierarchical shape task}

The mean Session 1 global shape score was $10.26(S D=$ 5.24), and the mean Session 2 global shape score was $11.48(S D=4.43)$ out of 16 . This global advantage was significant statistically in each session $(p \mathrm{~s}<.002)$ as compared to chance performance of 8 . The scores on this task ranged greatly across sessions, from 1 to 16 out of 16 .

\section{Global/local Navon letter task}

The mean letter identification RTs for Sessions 1 and 2 of the Navon letter task are presented in Fig. 5a and b, as a function of global or local task block and the congruence/ incongruence of global and local levels. Mean RTs were analyzed using a $2 \times 2$ (Task Block $\times$ Congruency) repeated measures ANOVA.

For both Sessions 1 and 2, we found a significant main effect of congruency, with faster RTs on congruent than on incongruent trials, $F(1,57)=101.98, p<.001$, and $F(1,57)=$ $108.69, p<.001$, respectively. We also found a significant main effect of task block in each session, such that the global trial RTs were faster than the local trial RTs, $F(1,57)=87.95$, $p<.001$, and $F(1,57)=228.20, p<.001$, respectively. Finally, a significant interaction emerged between task block and congruency in each session, such that the congruency effect was larger on local than on global trials, $F(1,57)=$ $10.78, p=.002$, and $F(1,57)=.25 .30, p<.001$, respectively. The finding that global information interfered more with local
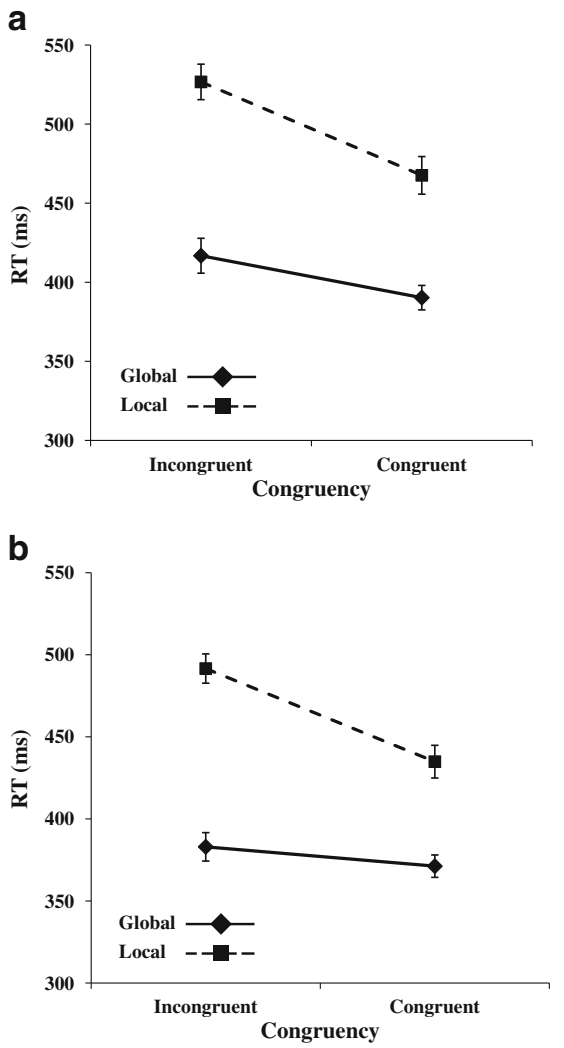

Fig. 5 (a) Mean Session 1 RTs on the Navon letter task, as a function of task (attend global or attend local) and target congruency. Error bars represent the standard errors for each condition mean. (b) Mean Session 2 RTs on the Navon letter task, as a function of task (attend global or attend local) and target congruency. Error bars represent the standard errors for each condition mean

responses than local information did with global responses provides evidence for a global advantage for this task. Across sessions, the average global interference scores ranged from -80 to $167 \mathrm{~ms}$.

The mean error rates in the Navon letter task were $8 \%$ and $7 \%$ for Sessions 1 and 2, respectively. A $2 \times 2$ (Congruency $\times$ Global/Local Task) repeated measures ANOVA on the mean error data for each session showed that errors were greater for incongruent than for congruent trials, $F(1,57)=36.46, p<.001$, and $F(1,57)=69.77$, $p<.001$, for Sessions 1 and 2, respectively. A main effect of task block was also apparent for Session 2 only, $F(1,57)=$ $7.55, p<.001$, as well as an interaction of task block and congruency for Session 2 only, $F(1,57)=14.54, p<.001$, in which the congruency effect was greater on local trials. This is consistent with the RT data, and thus does not suggest a speed-accuracy trade-off.

\section{Test-retest reliability}

As an index of test-retest reliability, the Pearson correlation coefficients between scores on Session 1 versus Session 2 
Table 3 Pearson zero-order correlations between test sessions for the three global-local measures (in bold font), as well as the relationships among all of the measures in Study 2

\begin{tabular}{llllll}
\hline & 1 & 2 & 3 & 4 & 5 \\
\hline
\end{tabular}

1. Global face, Session 1

2. Global shape, Session 1

.01

3. Global letter interference, Session $1-.02$

4. Global face, Session 2

$$
.57^{* *} .03-.07
$$

5. Global shape, Session 2

$\begin{array}{llll}-.10 & .64^{* *} & .04 & .03\end{array}$

6. Global letter interference, Session $2-.06$

$\begin{array}{llll}.19 & .27^{*} & -.10 & .07\end{array}$

${ }^{*} p<.05,{ }^{* *} p<.01$. Bold font indicates test-retest correlations

were examined individually for each of the three global/ local tasks, as in Study 1. Performance on the face global/ local task was shown to be reliable over time, as was that for the hierarchical shape global/local task (see the bolded values in Table 3, as well as Fig. 6a, b, and c). The test-retest reliability of the Navon letter task, however, was quite low, albeit significant. When we examined the mean Navon letter global and local RTs across sessions, both levels had high test-retest reliability ( $r=.66$ and .83 , respectively), suggesting that while the RTs were highly reliable, the measure of interference was less so, as in Study 1.

\section{Relationships among the measures}

The relationships among the three different measures of global/local processing bias were examined by correlating the scores for each test session. As in Study 1, none of the measures were significantly related to each other, either within a session or across sessions (see the nonbolded values in Table 3). ${ }^{5}$ When the scores for each of the three tasks were combined across the two sessions, once again we found no significant relationship among the three measures (see Fig. 7a, b, and c). This shows that once again, while the three global/local tasks were significantly reliable, they were unrelated to each other.

\section{Factor analysis}

As in Study 1, we performed a factor analysis with a varimax rotation that included our three measures of global bias for each of the two sessions (six items). Once again, we obtained a three-factor solution (see note 4), with each of the three global/local tasks loading on its own factor (see

\footnotetext{
${ }^{5}$ When performance on the Navon letter task was instead calculated as the difference between the mean local RTs and the mean global RTs, the same pattern of results was obtained, such that the test-retest reliability was still significant $(\mathrm{r}=.36)$ and the relationships among the three tasks were still nonsignificant (all ps $>.40$ ).
}
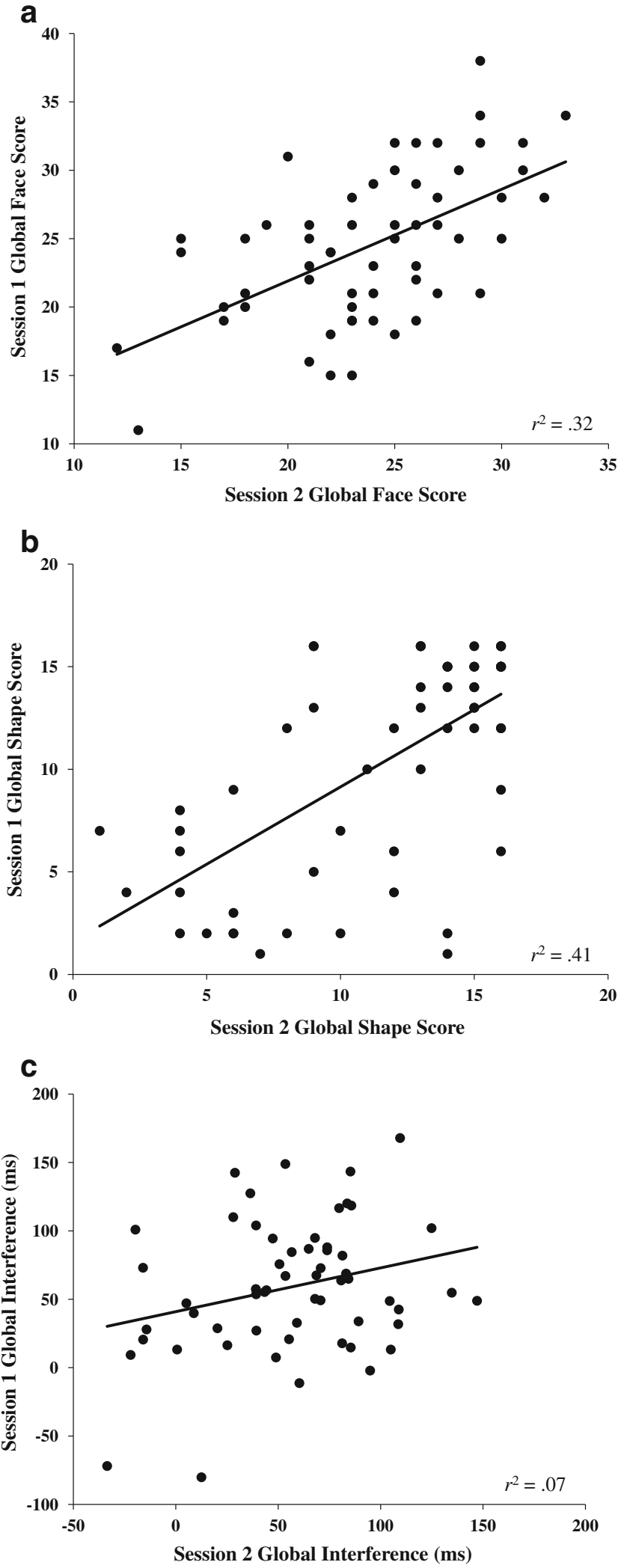

Fig. 6 (a) Scatterplot depicting Pearson $r$ correlations between Session 1 and 2 scores on the global/local face task. (b) Scatterplot depicting Pearson $r$ correlations between Session 1 and 2 scores on the global/local shape task. (c) Scatterplot depicting Pearson $r$ correlations between Session 1 and 2 scores on the global/local Navon letter task 

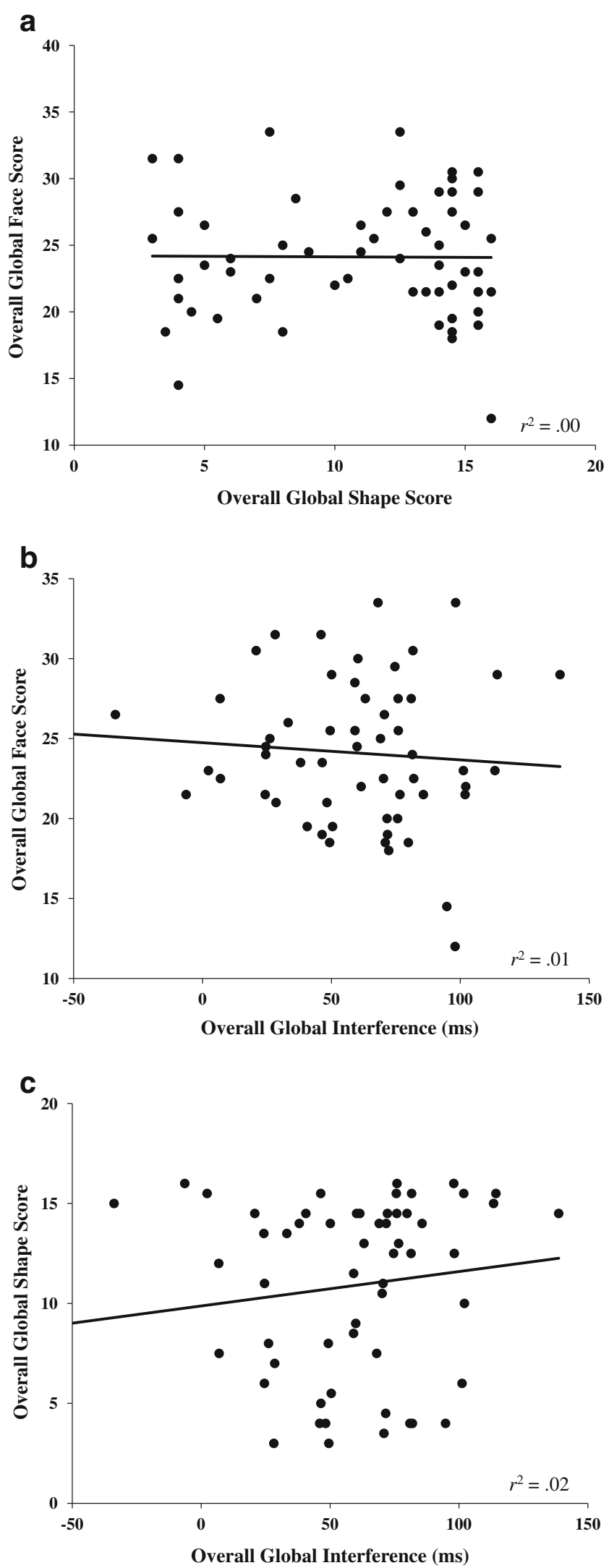

Table 4). This suggests that these three tasks were measuring unique processes.
Fig. 7 (a) Scatterplot depicting Pearson $r$ correlations between overall global face task score and overall global shape task score, $r=-.01$. (b) Pearson $r$ correlations between overall global face task score and overall global interference from the letter Navon task, $r=-.08$. (c) Pearson $r$ correlations between overall global shape task score and overall global interference from the letter Navon task, $r=.14$

\section{Discussion}

The purpose of Study 2 was to replicate the results of the first study, but using presentation conditions that promoted the typical global-advantage effect. In Study 2, all three of our tasks showed a typical global advantage, such that individuals were more globally biased. Individual performance on all three of our tasks was again significantly reliable over time. In general reliabilities were somewhat lower than in Study 1, but that would be expected, given that the greater global salience of the stimuli may have biased some individuals on at least some trials to be more globally biased than they might have been otherwise, thereby diluting the strength of the individual differences on these measures. Interestingly, the Navon letter task again had low, albeit significant, test-retest reliability, suggesting again that this task may not be a reliable measure of individual global/local preference.

Importantly, we once again found that while the results on our tasks were reliable, none of the tasks were related to each other. Indeed, the lack of relationships was very comparable to that observed in Study 1, and the factor analysis again showed that each of the three tasks loaded onto its own independent factor. As such, our findings from Study 1 cannot simply be the result of using stimuli that were too neutral or that were not measuring global/local processing, in the traditional sense.

\section{General discussion}

The primary purpose of this article was to examine the stability of individual global/local biases in two different

Table 4 Rotated factor loadings for the three global/local measures, by sessions, showing three distinct factors in Study 2

\begin{tabular}{lrrr}
\hline & \multicolumn{3}{l}{ Component } \\
\cline { 2 - 4 } & 1 & 2 & \multicolumn{1}{l}{3} \\
\hline Global face, Session 1 & -.06 & .89 & .02 \\
Global face, Session 2 & .06 & .88 & -.10 \\
Global shape, Session 1 & .90 & .04 & .09 \\
Global shape, Session 2 & .90 & -.04 & .04 \\
Global letter interference, Session 1 & .01 & -.01 & .81 \\
Global letter interference, Session 2 & .11 & -.07 & .78
\end{tabular}

Method: Principal components analysis, varimax with Kaiser normalization 
studies. In both studies, we found that the individual global/ local bias was stable over time, suggesting that individuals potentially develop a preference for processing global or local information, and that this preference persists over a period of at least several days. This, of course, does not suggest that bias is not influenced by the stimulus or task demands (e.g., Kimchi \& Palmer, 1982; Kinchla \& Wolfe, 1979) or participant state (e.g., Fredrickson \& Branigan, 2005; Gasper \& Clore, 2002), as this is well documented. It does, however, indicate that individuals may have a default processing strategy that influences their perception of visual objects.

Importantly, while the face task and both versions of the hierarchical shape task showed moderate-to-high test-retest reliability, the standard Navon letter task showed fairly poor reliability in both studies. It is unlikely that this occurred because RT was the dependent variable in the Navon letter task, as the RTs themselves were shown to be highly reliable over time, suggesting that the index of interference was what had poor reliability. The low reliability was also not the result of using a difference score as a measure of interference.

It is possible that the Navon letter task was less reliable because the other two tasks were forced choice tasks that pitted global versus local levels against each other on every trial, whereas the Navon letter task required the participant to follow instructions to direct attention to the local or the global level. This suggests that directing the level of attention may add noise, leading to lower task reliability. It is worth noting, however, that the Navon letter task is one of the most well-known and utilized tasks of global/local processing. The low test-retest reliability that we observed for this task suggests that caution should be taken when using this task as an individual-differences variable, and suggests the need for further investigation.

Our secondary purpose was to examine the relationships among three different measures of global/local processing. Unexpectedly, in both of our studies we found that none of the three global/local measures related to each other either within or across sessions, and that each loaded independently onto its own factor. This occurred despite the finding that all three tasks produced large individual differences and were reliable measures (with two being highly reliable).

Notably, the type of task used (level-directed speeded task or forced choice nonspeeded), the presentation mode (computerized or a paper-and-pencil task in Study 1), the nature of the stimuli (letters, shapes, or faces), and the timings of the stimulus presentation were different for some of the tasks. Additionally, letters tend to be perceived automatically as letters (Stroop, 1935), whereas the interpretation of hierarchical shapes or faces is thought to be more influenced by an individual's goals, motivations, or beliefs (e.g., Jemel, Pisani, Calabria, Crommelinck, \& Bruyer, 2003;
Langley, Laird, \& Rogers, 2009). Finally, it should be noted that the face task is not a common global/local task, and while spatial frequency is related to global/local processing, it is not global or local in and of itself. Therefore, it is possible that any or all of these large task differences could have resulted in the dissociability of these measures. It would be interesting to isolate these factors in order to determine which, if any, factors are critical for dissociating performance on these tasks. As such, we are currently conducting a series of studies to attempt to disentangle the critical factors responsible for the lack of differences among our tasks. What is clear is that if these three tasks are indeed measuring global/local processing, they are each measuring a unique aspect.

\section{Conclusion}

Global/local bias was found to be a reliable individualdifferences variable, especially when using the forced choice tasks employed here. However, although individual global/local bias on each of the tasks was reliable, the global/local bias on each task was unrelated to the bias on the other global/local tasks. Global/local tasks are often used interchangeably as indexes of global/local processing. This is somewhat alarming, as it suggests that researchers may be selecting a global/local measure on the basis of the ease of administration or the type of stimuli, without realizing that the task itself could have a large impact on their results. As such, we recommend caution when selecting a global/local task, particularly if comparing the results from two different global/local processing measures, as they may be measuring unique, rather than similar, processes.

Author note This work was funded by grants from the National Sciences and Engineering Research Council of Canada (NSERC), the Canadian Foundation for Innovation (CFI), and the Ontario Innovation Trust (OIT) to the second author. We thank Blaire Dube, Baris Bagcilar, and Tabitha Methot for assistance with the data collection.

\section{References}

Cattell, R. B. (1966). The scree test for the number of factors. Multivariate Behavioral Research, 1, 245-276. doi:10.1207/ s15327906mbr0102 10

Dale, G., \& Arnell, K. M. (2010). Individual differences in dispositional focus of attention predict attentional blink magnitude. Attention, Perception, \& Psychophysics, 72, 602-606. doi:10.3758/APP.72.3.602

Davidoff, J., Fonteneau, E., \& Fagot, J. (2008). Local and global processing: Observations from a remote culture. Cognition, 108, 702-709. doi:10.1016/j.cognition.2008.06.004

Deruelle, C., Rondan, C., Salle-Collemiche, X., Bastard-Rosset, D., \& Da Fonséca, D. (2008). Attention to low- and high-spatial frequencies in categorizing facial identities, emotions and gender in 
children with autism. Brain and Cognition, 66, 115-123. doi:10.1016/j.bandc.2007.06.001

Fink, G. R., Marshall, J. C., Halligan, P. W., Frith, C. D., Frackowiak, R. S. J., \& Dolan, R. J. (1997). Hemispheric specialization for global and local processing: The effect of stimulus category. Proceedings of the Royal Society B, 264, 487-494. doi:10.1098/ rspb.1997.0070

Fredrickson, B. L., \& Branigan, C. (2005). Positive emotions broaden the scope and attention and thought-action repertoires. Cognition and Emotion, 19, 313-332. doi:10.1080/02699930441000238

Gasper, K., \& Clore, G. L. (2002). Attending to the big picture: Mood and global versus local processing of visual information. Psychological Science, 13, 34-40. doi:10.1111/14679280.00406

Jemel, B., Pisani, M., Calabria, M., Crommelinck, M., \& Bruyer, R. (2003). Is the N170 for faces cognitively penetrable? Evidence from repetition priming of Mooney faces of familiar and unfamiliar persons. Cognitive Brain Research, 17, 431-446. doi:10.1016/ S0926-6410(03)00145-9

Kimchi, R. (1992). Primacy of wholistic processing and global/local paradigm: A critical review. Psychological Bulletin, 112, 24-38. doi:10.1037/0033-2909.112.1.24

Kimchi, R., \& Palmer, S. E. (1982). Form and texture in hierarchically constructed patterns. Journal of Experimental Psychology. Human Perception and Performance, 8, 521-535. doi:10.1037/ 0096-1523.8.4.521

Kinchla, R. A., \& Wolfe, J. M. (1979). The order of visual processing: "Top-down", "bottom-up", or "middle-out". Perception \& Psychophysics, 25, 225-231. doi:10.3758/BF03202991

Langley, P., Laird, J. E., \& Rogers, S. (2009). Cognitive architectures: Research issues and challenges. Cognitive Systems Research, 10, 141-160. doi:10.1016/j.cogsys.2006.07.004

Lux, S., Marshall, J. C., Thimm, M., \& Fink, G. R. (2008). Differential processing of hierarchical visual stimuli in young and older healthy adults: Implications for pathology. Cortex, 44, 21-28. doi:10.1016/j.cortex.2005.08.001

Martin, D., \& Macrae, C. N. (2010). Processing style and person recognition: Exploring the face inversion effect. Visual Cognition, 18, 161-170. doi:10.1080/13506280902868793

McKone, E., Davies, A. A., Fernando, D., Aalders, R., Leung, H., Wickramariyaratne, T., \& Platow, M. J. (2010). Asia has the global advantage: Race and visual attention. Vision Research, 50, 1540-1549. doi:10.1016/j.visres.2010.05.010

Minear, M., \& Park, D. C. (2004). A lifespan database of adult facial stimuli. Behavior Research Methods, Instruments, \& Computers, 36, 630-633. doi:10.3758/BF03206543

Navon, D. (1977). Forest before trees: The precedence of global features in visual perception. Cognitive Psychology, 9, 353-383. doi:10.1016/0010-0285(77)90012-3

Navon, D. (1981). The forest revisited: More on global precedence. Psychological Research, 43, 1-32. doi:10.1007/BF00309635

Nunnally, J. C. (1978). Psychometric theory (2nd ed.). New York: McGraw-Hill.

Paquet, L., \& Merikle, P. M. (1984). Global precedence: The effect of exposure duration. Canadian Journal of Psychology, 38, 45-53. doi: $10.1037 / \mathrm{h} 0080783$

Stoesz, B. M., Jakobson, L. S., Kilgour, A. R., \& Lewycky, S. T. (2007). Local processing advantage in musicians: Evidence from disembedding and constructional tasks. Music Perception, 25, 153-165. doi:10.1525/mp.2007.25.2

Stroop, J. R. (1935). Studies of interference in serial verbal reactions. Journal of Experimental Psychology, 18, 643-662. doi:10.1037/ h0054651

Yovel, G., Yovel, I., \& Levy, J. (2001). Hemispheric asymmetries for global and local visual perception: Effects of stimulus and task factors. Journal of Experimental Psychology. Human Perception and Performance, 27, 1369-1385. doi:10.1037/00961523.27.6.1369 